\title{
In-situ measurement technologies for flow behaviour and contact status of fibre-reinforced thermoplastics during forming with a six degree of freedom press
}

\author{
Birk Wonnenberg ${ }^{1, *}$, Franz Dietrich ${ }^{1}$, and Klaus Dröder ${ }^{1}$ \\ ${ }^{1}$ Institute of Machine Tools and Production Technology, Technische Universität Braunschweig, 38106 Braunschweig, Germany
}

\begin{abstract}
The paper presents investigations on a forming process, which is implemented in a forming press based on a Stewart platform. In contrast to common forming techniques, this buildup offers not only one but six degrees of freedom. This is of particular interest when it comes to the forming of materials that show significant anisotropic behaviour such as fibre-reinforced plastics. Therefore, an experimental setup is presented to record characteristic variables during the forming process of fibre-reinforced thermoplastics. The contact state is of particular interest for this kind of forming process because it changes continuously in shape and position as the forming process progresses. For this purpose, temperatures at different places in the tool are recorded to provide information about the flow velocity of the material and the contact state between tool and workpiece. This allows the determination of the exact time and position of the contact between material and forming tool as well as the duration of this contact. The results are compared with optical measurements analysed by image processing algorithm and process forces measured by load cells.
\end{abstract}

Keywords: Measurement, Press, Flow

\section{Introduction}

Forming processes with more than one degree of freedom in tool path have the ability to generate local material characteristics. An example for a machine, which can generate these forming processes and used in the experiments, is given in $[1,2]$. This machine is based on a Stewart platform, which has six degrees of freedom within the tool movement path. These additional degrees of freedom in the pressing movement provide a broad range of feasible process parameters. For instance, the tool geometry can be manufactured in shapes depending on the tool path, which result in different forming processes and material properties.

The anisotropic behaviour of certain materials is of particular interest for forming with a tool path more than one degree of freedom. It is proposed, that with the additional degree of freedom in the tool path a material specific forming process can generate beneficial properties in anisotropic materials. To generate these properties an understanding of the process is necessary and therefore a measurement technique to observe the process.

The scope of the paper is a measurement technique to detect the flow behaviour and contact state during forming. In particular, an in-situ measurement technique for hot forming of fibre-reinforced thermoplastic to detect the contact state between the material and the tool. The contact state has significant impact on the heat distribution and therefore on the viscosity of the material, which can be derived from the measurement.

To develop and test the measurement techniques, a simplified process within an experimental setup is needed. In the forming process, the material is pressed in a shape similar to a plate. The simplicity of this geometry facilitates process observation. The forming process is depicted in the progress from left to right in figure 1. In this process, the upper tool moves towards the material, pressing it onto the lower tool. During the process, the upper tool rotates around the tool centre point, so that a rolling movement is generated. This tool movement leads to a material flow, which should be observed in the forming process.

In the following sections, the material properties and sensor concepts in other scientific works are presented, followed by a description of the experiments.

\section{Material behaviour in the forming process and a proposal for a measurement technique}

In this section, the material properties are described. From this description, observable properties are derived and candidates for the measurement techniques are evaluated regarding the applicability in the experiments.

\footnotetext{
${ }^{*}$ Corresponding author: $\underline{b . w o n n e n b e r g @ \text { tu-braunschweig.de }}$
} 


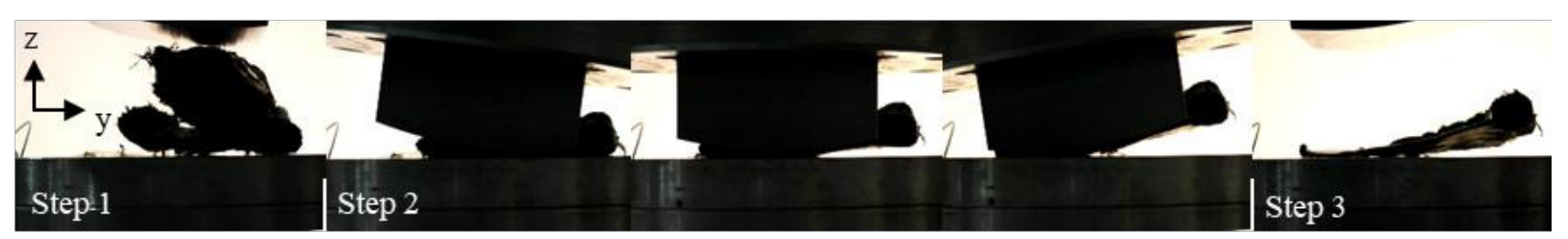

Fig. 1. Lateral view on the forming process in the progress from insertion of the material to finish (left to right)

The developed measurement technique used in the experiments shall help the understanding of the process. This understanding can provide a basis for an empirical description of the process. For the experiments, as example for an anisotropic material glass-fibrereinforced thermoplastic with a polypropylene matrix reinforced with a chopped fibre content of $30 \%$ was used.

In order to observe the process in the experiments, an understanding of the material is important; an overview is given in [3]. The flow behaviour and the material properties are directly related to the material temperature. Therefore, the heat change is of great importance processing the material. The material flow within forming tools is described in figure 2 [3]. While pressing, the material flow velocity has a gradient towards the middle of the cavity. As a consequence, warm material is transported from the middle to the surface. This process is dependent on the viscosity of the material, which is correlated with the temperature.

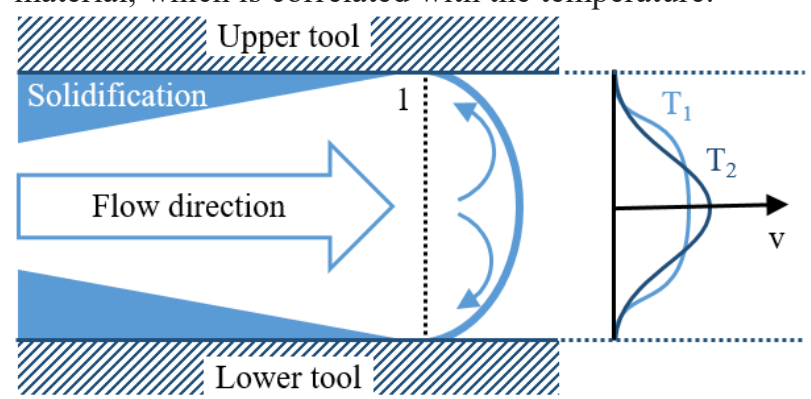

Fig. 2. Flow behaviour of molten thermoplastic in a forming process; (left) feeding current, flow front and solidification between the forming tools; (right) flow velocity profile at (1) in respect to temperature $\left(\mathrm{T}_{1}<\mathrm{T}_{2}\right)[3]$

From the information given in [3] the flow behaviour of the material in the forming process can be derived from the flow front and the heat flux to the tool. The work cited shows that it is possible to detect the flow front by presence of the material in a certain area optically, but not the contact state. The contact zone is not clearly defined in an optical perspective because of small gaps between the tool and the material. However, the contact zone can be determined by the heat flux to the tool. In a warm state, the matrix is melted and the material is soft and permeable. The heat flux from the material to the tool is relative low without pressure. If the forming process presses the material, it becomes solid and the heat flux rises.

With the description of the material, a measurement concept can be created. A starting point for the concept creation is the evaluation of other research work, which concerns the detection of material and the flow behaviour within forming processes.
In [4] closed-loop control of product properties in metal forming are reviewed by Polyblank et al. and an overview of sensors to observe the forming process are presented. From this overview thermocouples, load cells and a camera were chosen to observe the process, due to its simplicity of use. [4]

Apart from the use in other research work, the utilization of a camera in [5] to observe a forming process is a promising approach. Shimizu et al. [5] used a forming tool with transparent flank to observe the processes. In this work, the wear due to the contact between tool and sheet is examined. This setup from [5] could be the approach for the next attempt, if the experimental setup in this paper proves to be insufficient.

Hamedon et al. [6] used a borescope to observe the forming process, for instance sheet metal forming. The borescope was used in the process within the forming tool. However, in [6] this optical measurement was used to detect the edge of a bended metal sheet with a high contrast to the surrounding and a free line of sight within the forming tool.

\section{Experimental setup experimental procedure}

In this section, the experimental setup with the forming press, tool and sensors are developed and evaluated with a preliminary test. In the experiments, a material flow in a plain open forming tool was examined with regard to material state. The material state can be derived from the contact conditions between the material and the tool surface. While, the contact condition can be determined by the heat flux from the material into the tool. This shall reveal that the material state within the process can be determined by thermal sensors.

\subsection{Experimental setup}

The six degrees of freedom press used for the experiments is a hydraulically actuated Stewart platform. [1] The press is designed with a minimal cylindrical workspace of $100 \mathrm{~mm}$ in diameter and $50 \mathrm{~mm}$ in height and can rotate the tool $15^{\circ}$ around the $\mathrm{x}$ and $\mathrm{y}$-axis as well as $5^{\circ}$ around the $\mathrm{z}$-axis. The machine is designed to press with $50 \mathrm{kN}$ in $\mathrm{z}$-axis and $5 \mathrm{kN}$ in $\mathrm{x}$ - and $\mathrm{y}$-axis. Load cells are installed on each limp to record forces in the process.

The press was equipped with a tool, which was prepared with thermocouples. The positions of the thermocouples are based on the work of Yoneyama and Tozawa [7]. The thermocouples in [7] are placed in two different depth below the tool surface and measure the

$\overline{{ }^{*} \text { Corresponding author: b.wonnenberg} @ \text { tu-braunschweig.de }}$ 
temperature within the tool. With the tool temperature in two depth, the heat flux can be calculated. In addition, the surface temperature of the tool can be calculated with an inverse heat conduction.

The thermocouples are placed on and under the surface of the lower tool. Four sensors are inserted in holes from below. These holes vary in depth relatively to the upper surface of the lower tool. Sensors 3_2 and 4_2 are $2 \mathrm{~mm}$ and sensors $3 \_3$ and $4 \_3$ are $4 \mathrm{~mm}$ below the surface. Sensors 11 to 41 are placed on the tool surface, in top view sensor 31 is in line with sensors 32 and 33 , whereas sensor $\overline{4} 1$ is in line with sensors $4 \_2$ and 4_3. Sensors $1 \_1$ and $2 \_1$ are on the insertion area of the material. In figure $\overline{3}$, the position of the sensors is shown, sensor 2_1 is in the centre of the lower tool, sensor $1 \_1$ is $25 \mathrm{~mm}$ to the right side and sensors $3 \_1,3 \_2$ and $\overline{3} \_3$ are $5 \mathrm{~mm}$ to the left side, seen from the perspective of the camera. Sensors 4_1, 4_2 and 4_3 are $10 \mathrm{~mm}$ to the left side from the tool centre.
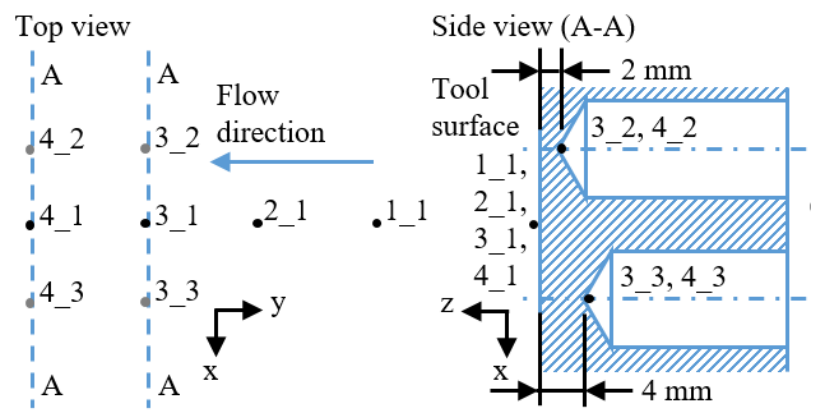

Fig. 3. Position of thermocouples; (left) top view with the flow direction of the material while pressing and position of sensors $1 \_1$ to $4 \_3$; (right) side view with the boreholes with $2 \mathrm{~mm}$ remaining material thickness above sensors 3_2 and 4_2 and $4 \mathrm{~mm}$ above sensors 3_3 and 4_3

Figure 4 shows the set up for a preliminary test of the sensors within a forming tool. The sensors were tested by applying ethylene-vinyl acetate (EVA) with a temperature of $160{ }^{\circ} \mathrm{C}$ on the tool surface. The EVA was applied in one step to get a synchronous heat input.
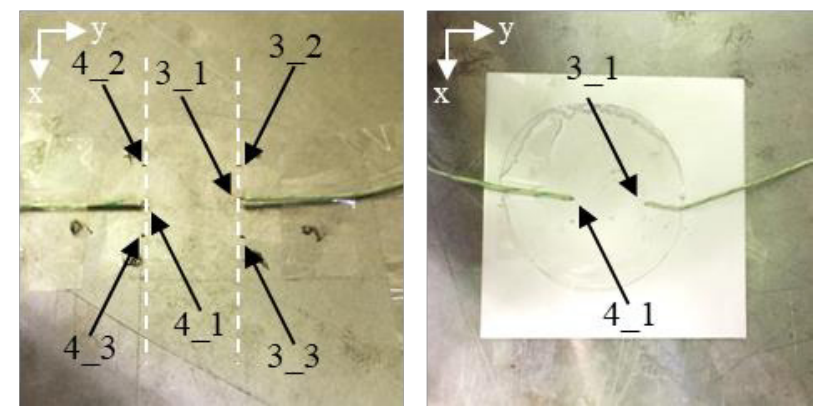

Fig. 4. Experimental setup for the sensor test, (left) the position of the sensors 3_1 to $3 \_3$ and $4 \_1$ to $4 \_3$ on the lower tool; (right) distribution of EVA after testing and removing from the tool surface

\subsection{Experimental procedure}

The experiment procedure was subdivided into a heating phase of the material, an insertion of the material into the press and a pressing phase. In the first phase, the material was melted in a convection oven at $220{ }^{\circ} \mathrm{C}$ for
10 min while the tools were kept at room temperature. In the second phase, the material was inserted into the press and on the surface of the lower tool. For the last phase, a pressing process was chosen with three forming steps. The first step is a vertical movement (z-axis) of the upper tool towards the material with a constant angle. In a second step, a rolling movement rotates the tool (Arotation axis) and in a third step the tool is lifted again. The other axis were kept constant without displacement over the process. The movement is displayed in figure 5 .

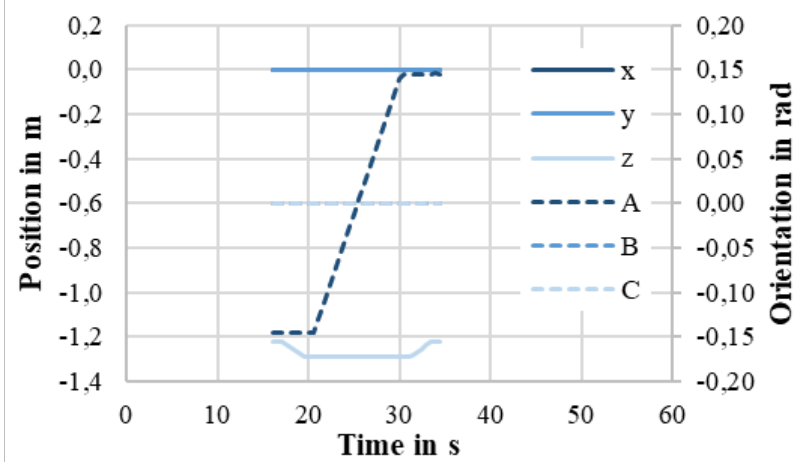

Fig. 5. Motion path of the forming tool calculated from internal position sensor readings of the press

In order to obtain diverse observations of the material flow, the experiments were separated into two cases. The cases vary according to the insertion position of the material into the process. The insertion position influences the forming results. In case A the material is placed on the centre of the forming tool during insertion. In case B the position of the material on the forming tool is shifted further to the right side. The positions have a huge impact on the flow behaviour in the forming process, which can be easily seen in figure 6 . Both figures show the process after the first forming step, in which the upper tool moves towards the lower tool in $\mathrm{z}$ axis. On the left side of figure 6 , the material is pressed towards the left side and fills the gap between upper and lower tool entirely. On the right side of figure 6 , which shows the case with the insertion position shifted to the right side, the material fills only three quarters of the same gap. After the first forming step in case A sensors $1 \_1$ to $4 \_1$ and in case $B$ sensors $1 \_1$ to $3 \_1$ are in contact with the material.

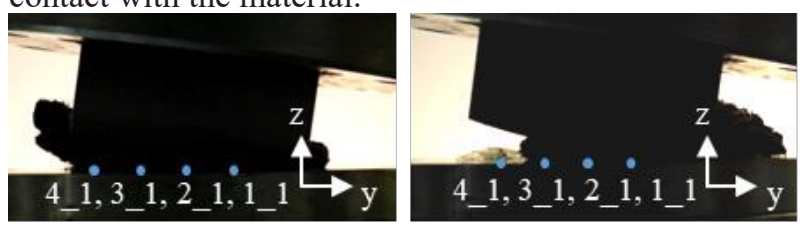

Fig. 6. Side view on the material distribution in case A (left) and case B (right) after the first forming step (tool movement in Z-axis); Sensors 1_1 to 4_1 are marked with points

\subsection{Image processing}

The process in the open tool was observed with a camera to detect the flow front of the material. In order to measure the positon of the flow front image processing algorithm were used to search for the furthest right and left edge of the material. The results from the image processing are displayed in figure 7 . An edge detection 
filters the tool and material surface. By shape recognition, the upper and lower tool are detected and removed from the image. The remainder is the material in the forming process, coloured in blue in figure 7 . The farthest left (1) and right (2) edge are detected and the positions are plotted in relation to the lower tool centre.

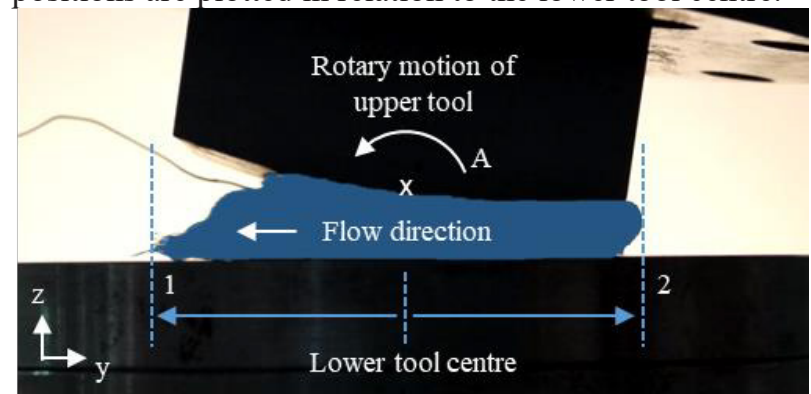

Fig. 7. Observation of a hot forming process with superimposed rolling motion (A) in the open die (moulding compound is coloured in blue after the detection in the image processing); (1) is the farthest left and (2) the farthest right edge of the material measured from the lower tool centre

\section{Results}

This section describes the results of the preliminary test and the measurements of case A and B. The temperature, force and the results from the image processing are displayed in figure 9. Distinctive points in the graphs are marked by numbers to simplify the discussion of the results. The results show a correspondence between heat distribution within the tool and the material distribution on the surface of the tool, measured by thermocouples and a camera with image processing.

\subsection{Preliminary test}

In figure 8 , the temperature profiles of the preliminary test are displayed. With the application of EVA on the surface of the tool, the temperature increases at each sensor. At (1) the profile of sensors 3_2, 3_3, 4_2 and 4_3 rise nearly instantaneously after the increase at sensors $3 \_1$ and $4 \_1$ but with a smaller gradient. Sensors 31 and $\overline{4} 1$ are attached to the surface of the lower tool and thus reach the highest values at (3), 98 and $78{ }^{\circ} \mathrm{C}$. The peak values of sensors $3 \_2,3 \_3,4 \_$and $4 \_3$ are 25 , 24,26 and $23{ }^{\circ} \mathrm{C} .10 \mathrm{~s}$ after the application at $(\overline{2})$ sensors 3 2, 3 3,4 2 and $4 \_3$ show the respectively same spread as before the application.

\subsection{Results from case $A$ and $B$}

The results of the experiments are displayed in figure 9 . Figure 9 displays six diagrams and is separated in two columns for cases A and B. Diagrams a) and b) show the thermal measurement of cases A and B respectively. Diagrams c) and d) show the forces measured in cases A and B. Diagrams e) and f) show results from the image processing with the location and velocity of the flow front in cases A and B.
The time scale of the following diagrams are adjusted and synchronised, so that at $20 \mathrm{~s}$ the first forming step is finished and at $30 \mathrm{~s}$ the second forming step is finished.

For case A, diagram a) shows the temperature profile. The initial temperature for all sensors is $19{ }^{\circ} \mathrm{C}$. Sensors 1_1, 2_1 and 3_1 detect a first increase (1a) of the temperature with the insertion of the material. The second increase (4a) is caused by the first forming step, when the material is compacted over sensors 1_1, 2_1 and 31 . Sensor 41 was not covered by the material during insertion, but the first increase of the temperature is induced by the first forming step, which can be explained by the left side of figure 6. Material is pushed onto the section where sensor 41 is attached which leads to the temperature increase that is measured by sensor 4 1. The sensors under the surface of the tool $\left(3 \_2,3 \_3,4 \_2,4 \_3\right)$ detect the first temperature increase directly after the first forming step.

The measured peak temperature in case $\mathrm{A}$ are in order 2_1, 1_1, 3_1, 4_1 for the surface sensors, starting at $56{ }^{\circ} \overline{\mathrm{C}}$ for sensor $2 \overline{1}_{1}(4 \mathrm{a}), 65^{\circ} \mathrm{C}$ for sensor $1 \_1(5 \mathrm{a})$, $77{ }^{\circ} \mathrm{C}$ for sensor 3_1 (6a) to $117{ }^{\circ} \mathrm{C}$ for sensor 4_1 (7a). Under the surface, sensors 3_2, 3 3, 4_2 and 4_3 have

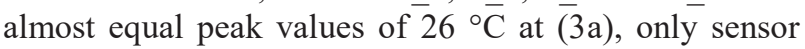
$4 \_2$ detects $25^{\circ} \mathrm{C}$.

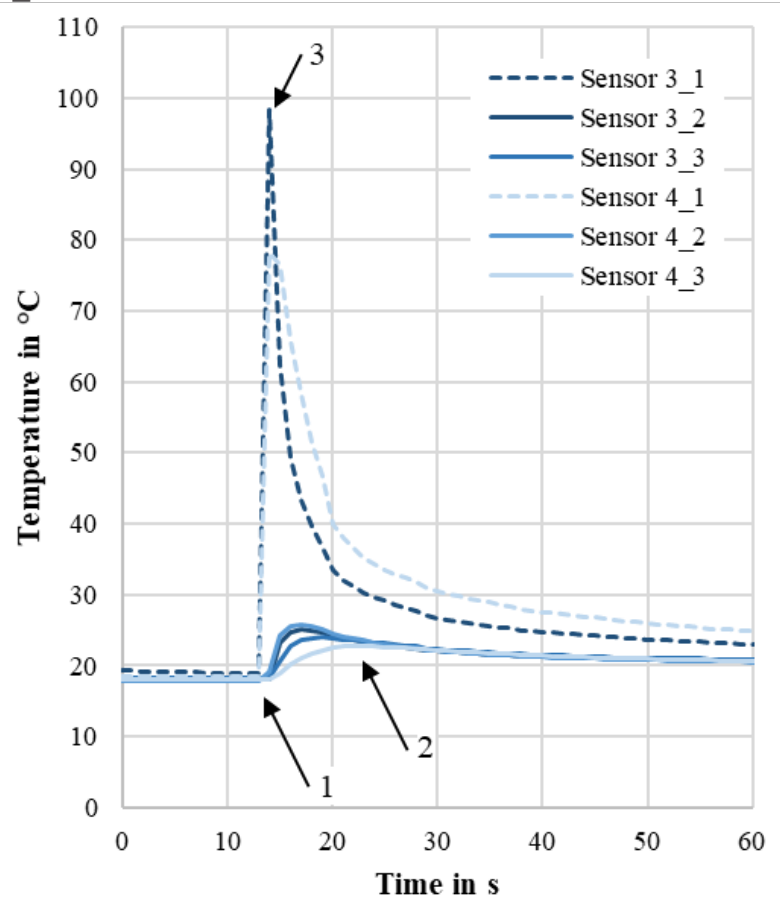

Fig. 8. Temperature profile of the sensors tested with EVA heated to $160{ }^{\circ} \mathrm{C}$; at (1) the measured temperature of sensors $3 \_1$ and $4 \_1$ rise to a peak temperature of 98 and $78{ }^{\circ} \mathrm{C}$; between (1) and (2) sensors 3_2, 3_3, 4_2 and 4_3 have a peak at $25,24,26$ and $23^{\circ} \mathrm{C}$

In case B the measured temperatures are presented in diagram b) of figure 9. The initial temperature for all sensors is $20^{\circ} \mathrm{C}$. Sensors $1 \_1$ and $2 \_1$ detect the first temperature increase with the insertion of the material (1b) and the second after the first forming step (5b). In case B sensor 3_1 measures a temperature increase after the first forming step (2b). Sensor 4_1 detects the first rise at the end of the second forming step (3b). 

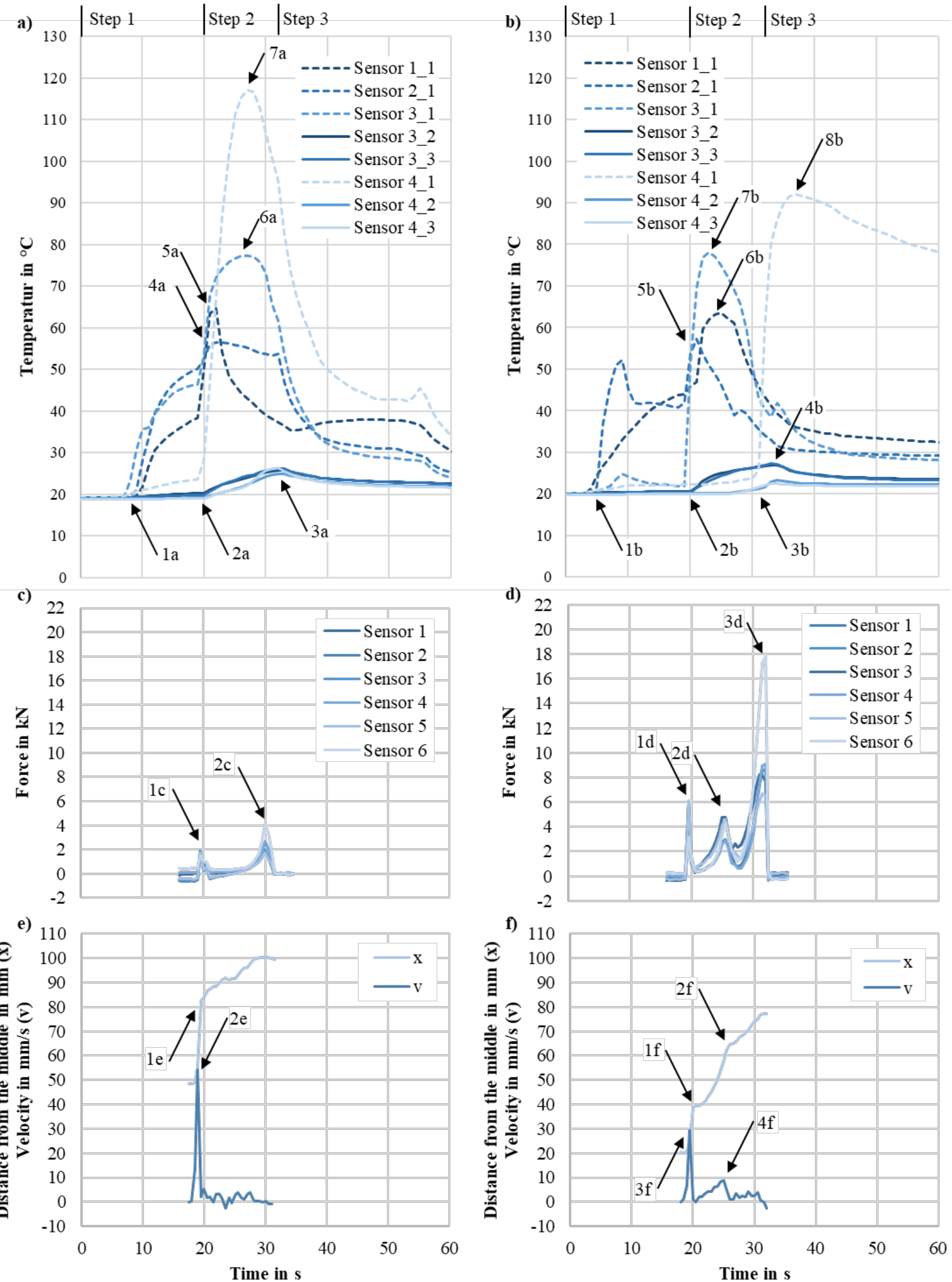

Fig. 9. a) Temperature profile from case A, all sensors are covered with material after the first tool movement in $z$-axis (2a), peak temperature of sensors $1 \_1$ to $4 \_3: 65,56,77,26,26,117,25,26{ }^{\circ} \mathrm{C}$ (3a to 7a); b) Temperature profile from case B, only sensors $1 \_1$ to $3 \_3$ are covered with material after the first tool movement in z-axis (2b), peak temperature of sensors $1 \_1$ to $4 \_3: 63,57,78,27$, $27,92,23,22{ }^{\circ} \mathrm{C}$ ( $4 \mathrm{~b}$ to 8 b); c) Case A, force signals from load cells above the upper tool; d) Case B, force signals from load cells above the upper tool; e) Movement of the flow front to the left side measured optically from the centre of the lower tool in case A; f) Movement of the flow front to the left side measured optically from the centre of the lower tool in case B 
Similar to sensor 3_1, sensors 3_2 and 3_3 measure the first increase of the temperature under the surface after the first forming step (2b). After the second forming step sensors $4 \_2$ and $4 \_3$ detect a temperature rise concurrent with the surface sensor $4 \_1$ at (3b). The measured temperature increases after the first step, which matches with the right side of figure 6 . With the first step, material is pushed onto sensor $1 \_1$ to 31 , while the section with sensor $4 \_1$ is still without contact to the material.

The peak temperature in case $\mathrm{B}$ are in order $2 \_1,1 \_1$, $3 \_1,4 \_1$ for the surface sensors, were sensor 2_1 has a peak at $57^{\circ} \mathrm{C}(5 \mathrm{~b})$, sensor $1 \_1$ at $63{ }^{\circ} \mathrm{C}(6 \mathrm{~b})$, sensor $3 \_1$ at $78{ }^{\circ} \mathrm{C}(7 \mathrm{~b})$ and sensor $4 \overline{1}$ has a peak at $92{ }^{\circ} \mathrm{C}(8 \bar{b})$. Under the surface, sensors $3 \_2$ and 3 _ 3 show almost equal temperatures at $27{ }^{\circ} \mathrm{C}(\overline{4 b})$ and $4 \overline{2}$ and 43 have lower peak values at $23{ }^{\circ} \mathrm{C}$ and $22{ }^{\circ} \mathrm{C}(4 \mathrm{~b})$.

In parts c) and d) in figure 9 the force signals from the load cells are displayed. Two force peaks were measured at the end of the first (1c, 1d) and second forming step (2c, 3d). Case B exhibits a third force peak in the middle the second forming step (2d). This is due to a movement of the upper tool, which was adjusted with a small step in z-direction towards the lower tool to achieve a constant material thickness.

Cases A and B were observed with a camera. With an image processing, the flow front in these experiments was measured optically. The results of this measurement are presented in the following section.

In case $\mathrm{A}$, the left edge of the material located at about $50 \mathrm{~mm}$ left from the centre of the lower tool after the insertion. With the first forming step the left flow front moved to $88 \mathrm{~mm}$ (1e) with a peak velocity of about $50 \mathrm{~mm} / \mathrm{s}(2 \mathrm{e})$. Due to the second forming step the flow front moved to $100 \mathrm{~mm}$.

In case $\mathrm{B}$, the material was only $20 \mathrm{~mm}$ left from the lower tool centre after insertion. The variant position of the material after insertion was part of the experimental design. After the first forming step, the flow front moved to $39 \mathrm{~mm}(1 \mathrm{f})$ with a peak velocity of about $30 \mathrm{~mm} / \mathrm{s}$ (3f). In the middle of second forming step, another peak in the velocity of about $10 \mathrm{~mm} / \mathrm{s}(2 \mathrm{f}, 4 \mathrm{f})$ was detected. After the second forming step, the flow front was $77 \mathrm{~mm}$ left from the lower tool centre.

\section{Discussion}

The discussion sets the results of the measurements into context with each other. Furthermore, the results are compared to literature.

The preliminary test showed that thermocouples on and under the surface are able to detect increases of the surface temperature at the same time. The peak temperature between a sensor on the surface and under the surface differ between 52 to $75{ }^{\circ} \mathrm{C}$. In [7] the surface temperature was calculated by using the inverse heat conduction problem using two thermocouples in two different depth. In the experiments, conducted in [7], the specimen was made out of aluminium and had a higher initial temperature. The outcome is a steep temperature gradient in the tool. In this paper, the signal difference,
$3{ }^{\circ} \mathrm{C}$, in preliminary test is too little, in respect to the measurement inaccuracy, $\pm 1,5^{\circ} \mathrm{C}$ form the thermocouple type $\mathrm{K}$ class 1 [8] and $\pm 0,5{ }^{\circ} \mathrm{C}$ form the measuring instrument, to calculate a surface temperature. The same applies to the case A and case B, see figure 9 ( $3 a, 3 b$, 4b). To calculate the surface temperature of the tool surface or the heat flux the measurement accuracy has to be higher.

The contact state between material and tool in the forming process can be detected by thermocouples. Even sensors under the surface of the tool can detected a temperature increase, if warm material is pressed on the tool surface during the forming process. Material, which only lays on the surface and has no contact to the upper tool, does interfere with thermocouples on the surface but not with those under the surface. This is due to the pressure dependent thermal conductance between the material and the tool. In figure 9 diagram d) displays the amount of pressing force used in case B. At (2d) the pressing force has a peak, which has no influence on the temperature profile of sensors under the surface ( $3 \_2$ to 4 3) between $(2 b)$ and $(3 b, 4 b)$. This indicates that, after a compaction a contact between material and tool is enough for a detectable heat flow, while the amount of pressing force has no significant influence on the detection of the temperature increase or absolute value.

In both cases $\mathrm{A}$ and $\mathrm{B}$ a highest temperature peak was measured with surface sensor $4 \_1(7 a, 8 b)$, which is further away from the insertion area than sensors 1_1 to 3 1. This can be explained by the flow behaviour of the material, see figure 2. While pressing warm material flows from the core to the surface. Although the surface temperature is higher, the measured tool temperature at this point is colder ( $3 \mathrm{~b}$ ) than close to the insertion area (4b). This leads to the conclusion that measuring the temperature under the tool surface with thermocouples cannot give significant data about the material temperature without knowing the distribution of the material and the heat distribution within the tool at this point. The material distribution on the other hand can be determined with thermocouples under the tool surface.

In figure 9 diagram c) and d) display the profile of the process force measured by the load cells. The load cells show peak values at specific points in the pressing process. At the end of the first (1c, 1d) and second step $(2 \mathrm{c}, 3 \mathrm{~d})$ a peak was detected. For itself, the measurement is not significant enough to interpret the flow behaviour, as the reaction force is influenced by other parameters, for instance the material viscosity. However, these values can be compared to simulations and therefore should be recorded for further studies.

With the help of figure 9 diagrams e) and f) it can be shown, that the insertion position of the material has a huge impact on the material flow during the forming process with a six-degree of freedom tool path. The initial positions differ $30 \mathrm{~mm}$ from each other. This difference leads to an almost filled gap between the upper and the lower tool in case A after the first forming step (1e). In contrast to this, the gap between the forming tools in case B is only filled after the second forming step due to the different material flow (2f). The progress of the material flow in cases A and B corresponds with 
the temperature profiles of the thermocouples under the surface. Therefore, it is possible to detect the flow front in the process with the thermocouples.

With the presented measurement technique, the forming process and the flow of the material can be observed. The observations show that the results of the forming process depend strongly on the flow behaviour of the material and therefore on the location of the material insertion.

\section{Conclusion}

Hot forming with a six degree of freedom forming press provides a broader range of process parameters than linear pressing movements. For instance, the tool motion path can induce shearing in the material or localised pressure conditions. Furthermore, the forming tools can be manufactured with less constraints concerning the geometrical freedom. The combined influence of the tool geometry and the motion path on the forming process can direct the flow of the material. The forming result depends on the insertion position of the material and the specific material flow in each procedure.

To determine the material state and the flow direction in the forming process, it is proposed to use the flow front and the contact state of the material in certain areas. To detect the flow front and the contact state measurement techniques are necessary and an experimental setup is developed for these techniques. The purpose measuring techniques are tested concerning the detection of the flow front and contact state of the material within the tool.

The basis of the experimental set up was a six degree of freedom forming press. The press was equipped with an open tool to observe the process optically. In addition, the tool was equipped with thermocouples on and under the surface. With the help of these thermocouples, the heat from the hot forming of fibre-reinforced thermoplastic was measured. The experiment is split into two cases with different insertion places of the material and thus different flow behaviour to demonstrate the usability of the measurement technique.

Thermocouples and load cells are utilized to observe the process state. In contrast to the presented experiments, an optical observation in closed tools is difficult realize, as long as the tool is not transparent.

With the presence of warm material pressed on the surface of the tool an increase of the temperature can be detected with thermocouples. For this purpose, the thermocouples can be positioned on or under the tool surface. The load cell measurements allow the recording of the process force and the detection of specific forming steps in the process. The presence of the material in a certain area can be captured optically with a camera. With image processing, the flow front can be detected and the flow velocity can be calculated.

Thus, in this state, the measuring technique with thermocouples is not suitable for closed loop control of the process, yet. The acquired information is a punctual measurement with a signal that only has two states: contact and no contact. For continuous control of the process this measurement techniques must be enhanced in resolution and significance of the signal, which will be the subject of further research.

The distance between thermocouples and the surface of the tool has little effect on the measured temperature. In this process with these parameters, it is not possible to calculate neither the heat flux nor a reliable estimation of the surface temperature. Therefore, the depth of two thermocouples must differ more widely or a higher measurement accuracy is needed for this experimental setup.

As a concluding remark, this measurement technique allows to detect the flow front of a warm material within the forming tool. The information can be used as a reference for simulations or for the observation of the forming process in production for quality reasons. The information from a punctual measurement has a lower resolution than an optical measurement, but it can be utilized in closed tools and give an information whether the material is in direct contact or not, which is difficult to determine optically.

This work was supported by the German Research Foundation (DFG DR 973/4-1)

\section{References}

1. J. Hesselbach, B.-A. Behrens, F. Dietrich, S. Rathmann, J. Poelmeyer, Prod. Eng. Res. Devel., Flexible forming with hexapods, 1 429-436 (2007)

2. F. Dietrich, Non - linear modelling of hydraulically actuated production machines using optimised experiments (Vulkan, Essen, 2014)

3. A. Radtke, Steifigkeitsberechnung von diskontinuierlich faserverstärkten Thermoplasten auf der Basis von Faserorientierungs- und Faserlängenverteilungen (Fraunhofer IRB-Verl., Stuttgart, 2009)

4. J.A. Polyblank, J.M. Allwood, S.R. Duncan, J. Mater. Process. Technol., Closed-loop control of product properties in metal forming: A review and prospectus, $2142333-2348$ (2014)

5. T. Shimizu, T. Kakegawa, M. Yang, Y. Qin, T.A. Dean, J. Lin, S.J. Yuan, F. Vollertsen, MATEC web conf., Development of in-situ observation system of dynamic contact interface between dies and materials during microforming operation, 21 9008 (2015)

6. Z. Hamedon, K. Mori, Y. Abe, J. Mater. Process. Technol., In-situ measurement of three-dimensional deformation behaviour of sheet and tools during stamping using borescope, 214 945-950 (2014)

7. T. Yoneyama, Y. Tozawa, CIRP Ann. Manuf. Technol., Direct Measurement of Stress and Heat between Work and Tool in Metal Forming, 39 219222 (1990)

8. DIN EN 60584-1, Thermoelemente - Teil 1: Thermospannungen und Grenzabweichungen (IEC 60584-1:2013), (2014) 